\title{
Improvement of Field Accuracy and Plasma Performance in the RT-1 Device
}

\author{
Yoshihisa YANO, Zensho YOSHIDA, Junji MORIKAWA, Haruhiko SAITOH, Hiroyuki HAYASHI \\ and Tatsunori MIZUSHIMA \\ Department of Advanced Energy, University of Tokyo, Kashiwa, Chiba 277-8561, Japan
}

(Received 19 June 2009 / Accepted 3 July 2009)

\begin{abstract}
To improve the accuracy of the magnetic field of Ring Trap-1 (RT-1), we have constructed a system of correction coils to cancel the geomagnetic field and control the attitude of the floating magnet. Without the geomagnetic field canccellation, the floating magnet tilts about 1.4 degrees. The previous prototype correction coils have been replaced by new coils that are much larger and farther from the chamber, so the error field due to the multipole components of the correction field is reduced by a factor of 30 (from $2.6 \%$ to $0.1 \%$ of the confinement field near the edge region). A significant improvement in plasma confinement has been observed (the stored energy of the plasma has been increased by a factor of 1.5).
\end{abstract}

(c) 2009 The Japan Society of Plasma Science and Nuclear Fusion Research

Keywords: levitated dipole confinement, field accuracy, geomagnetic, correction coils, high-beta plasma

DOI: $10.1585 /$ pfr.4.039

The Ring Trap-1 (RT-1) device has a magnetospheric confinement field generated primarily by a superconducting ring magnet floating in a vacuum chamber $[1,2]$. The lifting coil is located at the top of the chamber. The motion of the magnet has six degrees of freedom consisting of the position of the center of the mass $(x, y, z)$, the attitude $\left(\theta_{x}, \theta_{y}\right)$ and the rotation $\theta_{z}$. In RT- 1 , the magnet is unstable with respect to vertical $(z)$ motion, while it is stable with respect to sliding $(x, y)$ and tilting $\left(\theta_{x}, \theta_{y}\right)$ motions. We suppress the vertical instability of the magnet by a PID feedback control system, and regulate the displacement in $z$ within $0.1 \mathrm{~mm}$. Although the tilting motion is stable, small nonuniformities of the mass distribution of the ring magnet as well as the geomagnetic field yield a finite tilt and slow oscillations of the attitude. The tilt due to the mass unbalance is reduced to less than 0.05 degrees (from 0.88 degrees initially) by adjusting the mass distribution. The aim of this development is to minimize the attitude-angle of the floating magnet and improve the plasma confinement by canceling the geomagnetic field with a sufficiently small error field.

The RT-1 experiment began operation with smaller prototype correction coils that were installed near the chamber. Using these coils, we tested the attitude control and proved that the magnet can be made level when we apply a $0.3 \mathrm{G}$ horizontal magnetic field, which just cancels the horizontal component of the geomagnetic field in Eastern Japan [3]. The new and prototype correction coils are compared in Figs. 1 and 2. The correction coils are composed of two Helmholtz coils crossing at right angles. The prototype coils are located at $r=1.4 \mathrm{~m}$ from the center of

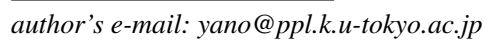

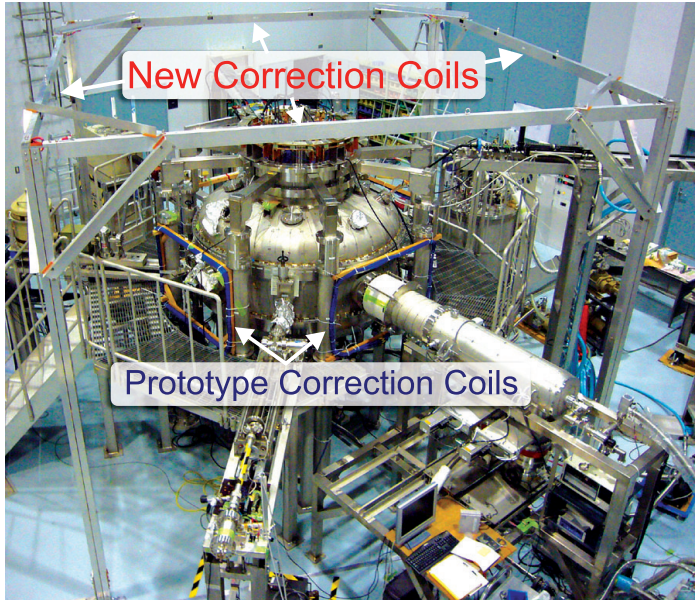

Fig. 1 Photograph of the RT-1 device and the correction coils. The prototype coils have dimensions of $1.2 \mathrm{~m} \times 0.8 \mathrm{~m}$. The new correction coils are made of aluminum selfstanding frames with a measurements of $4.5 \mathrm{~m} \times 4.5 \mathrm{~m}$ $\times 4.0 \mathrm{~m}$.

the chamber (about $0.5 \mathrm{~m}$ from the edge of the plasma region) so the error field (multipole component produced by the correction coils) is considerably strong. The new system of correction coils was designed to reduce the error field less than $0.1 \%$ of the mean field at the edge region. Numerical estimates of the error fields of both correction coils are shown in Fig. 3, where we assume a $0.3 \mathrm{G}$ geomagnetic field to the north; the correction coil current is set to make the average field at $r=0.25 \mathrm{~m}$ (magnet position) to cancel the geomagnetic field. The prototype coils produce a maximum error field of about $1.3 \mathrm{G}$, which is 


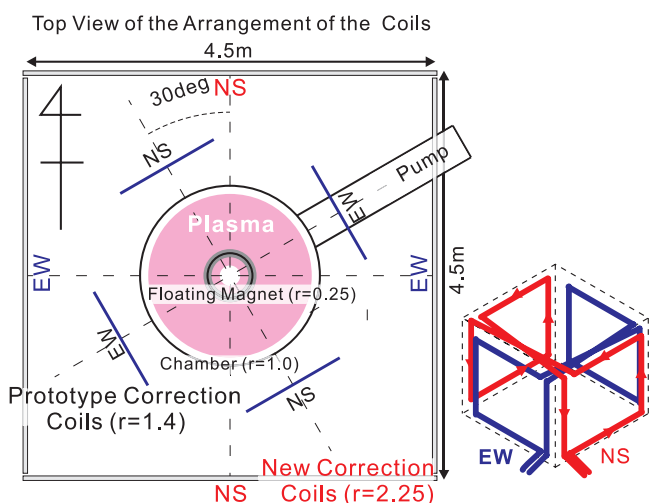

Fig. 2 The arrangement of the RT- 1 device and the correction coils. The prototype coils are not parallel to the geomagnetic field, while the new coils are set approximately parallel to the geomagnetic field. The EW coils are installed for the fine adjustment of the magnetic field.

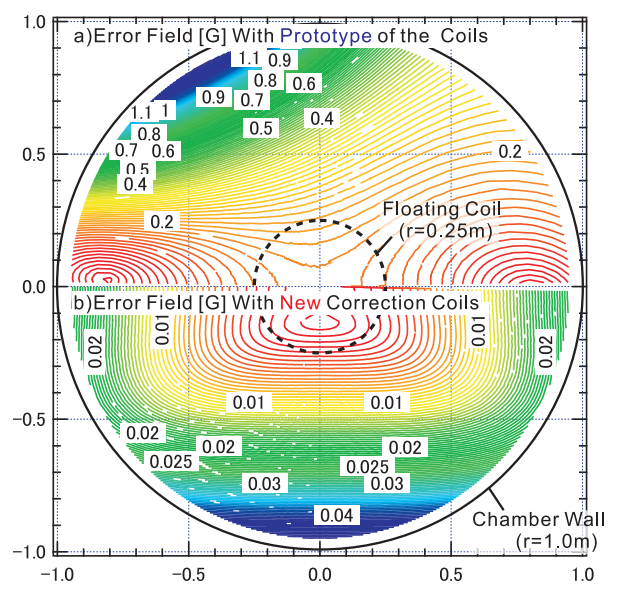

Fig. 3 Calculation results of the strength of the error field caused by the multipole component of (a) the prototype (upper figure) and (b) the new correction coils (lower figure). The distribution of the field is shown on the mid-plane $(z$ $=0$ ). Field strength is given in gauss.

$2.6 \%$ of the ambient field at $r=1.0 \mathrm{~m}$. In contrast, the new coils reduce the maximum error field to $0.05 \mathrm{G}$, which is $0.1 \%$ of the ambient field.

A typical experimental result for the attitude control is shown in Fig. 4. The magnet rotates like a pendulum, because the heaviest point oscillates around the minimum potential of the error field (see Fig. 4). Without attitude control, three vertical positions (P1, P2, P3) of the magnet (measured by laser sensors) have appreciable differences, which correspond to about 1.4 degrees of tilt of the magnet. When we apply the correction field, the tilt is reduced to less than 0.05degrees.

With the new correction coils, we have observed appreciable improvement of the plasma confinement. The diamagnetic signals have been increased more than $10 \%$ in comparison with the previous data with the old prototype

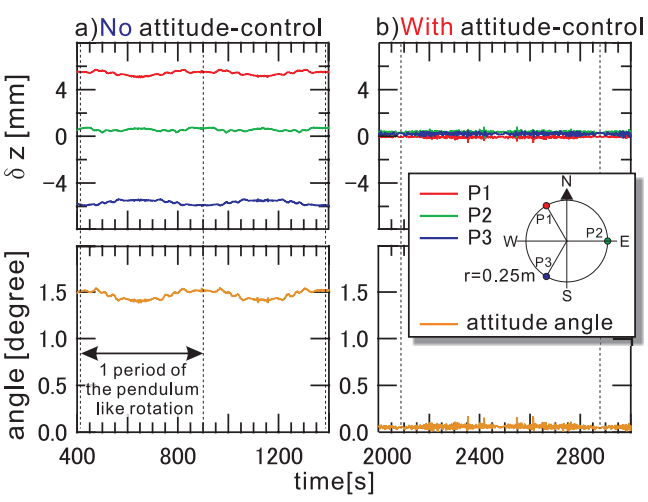

Fig. 4 Demonstration of attitude control. The vertical position of the floating magnet is measured at three points (P1, $\mathrm{P} 2, \mathrm{P} 3$ ) on the upper surface of the coil casing (shown in upper figures). The tilt angle is estimated by the differences among these three positions (shown in lower figures). In these plots, the signals have been averaged over 30 seconds to reduce high-frequency fluctuations caused by micro-scratches on the magnet's surface. (a) Left: Without the correction field, the magnet is tilted about 1.4 degrees. (b) Right: With the optimum correction field, the tilt is decreased to 0.05 degrees.

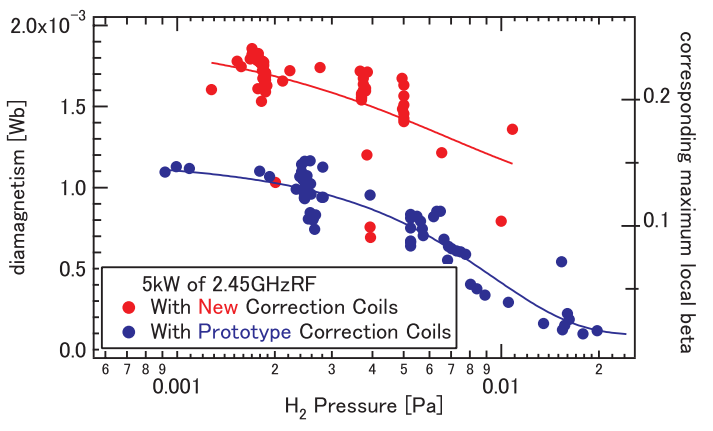

Fig. 5 Comparison of the diamagnetic signals for plasmas with the prototype and new correction coils. The diamagnetic signal of $1 \mathrm{mWb}$ corresponds to about 0.13 of the local beta [4]. The diamagnetic signal in RT-1 depends mainly on the filling gas pressure and the heating power, and it has a large variation when we take the pressure on the horizontal axis. The lower diamagnetic signals with the new correction coils in this graph are measured at the beginning of the experiment, when outgassing from the chamber wall is thought to be large.

correction coils [4]. The improvement is more significant in operation with a lower RF power (Fig. 5). The diamagnetic signal is enhanced by a factor of 1.5 in plasma with $5 \mathrm{~kW}$ RF power.

In conclusion, we have succeeded in improving the plasma confinement of RT-1 by constructing new fieldcorrection coils and reducing the field error.

[1] Z. Yoshida et al., Plasma Fusion Res. 1, 008 (2006).

[2] Y. Ogawa et al., Plasma Fusion Res. 4, 020 (2009). 
[3] Kakioka magnetic observatory Japan meteorological agency website, http://www.kakioka-jma.go.jp/en/index.html

[4] Z. Yoshida et al., High-Beta (Hot Electron) Plasma in
Ring Trap 1 (RT-1), 22th IAEA Fusion Energy Conference, Switzerland, 2008, EX/P5-28. 